\title{
Acceso de las mujeres a los cargos directivos: universidades con techo de cristal ${ }^{*}$
}

\author{
Women's access to management positions: universities \\ with glass ceiling
}

Acesso das mulheres aos cargos diretivos: universidades

com teto de cristal

\footnotetext{
${ }^{*}$ Chileno, desde 1998 es académico del Departamento de Ciencias Sociales de la Facultad de Ciencias Sociales, Artes y Humanidades de la Universidad de Antofagasta en Chile. Doctor en Procesos de Cambios Social (2011) Universidad de Valladolid-España, investigador de la temática de responsabilidad social de las organizaciones. ORCID: oooo-ooo32359-2304. Correo electrónico: ricardo.gaete@uantof.cl

** El artículo es resultado del proyecto de investigación denominado «Acceso de las mujeres a los cargos directivos: universidades con techo de cristal. Un estudio de caso», desarrollado entre los años 2016 y 2017, código AED 15-16-04 financiado por la Vicerrectoría de Investigación, Innovación y Postgrado de la Universidad de Antofagasta-Chile, a través del Programa de Fomento a la investigación en áreas escasamente desarrolladas. Artículo de investigación recibido el 16.06.2017 y aceptado el 28.11.2017.
} 


\section{Cómo citar}

GAETE QUeZADA, R. (2018). Acceso de las mujeres a los cargos directivos: universidades con techo de cristal. Revista CS, (24), 67-90.

DOI: http://dx.doi.org/10.18046/recs.i24.2431. 
Resumen

Abstract

Resumo

Se analiza el techo de cristal desde las percepciones y experiencias de las académicas respecto al acceso a los cargos directivos en sus instituciones universitarias, materializado en barreras internas o externas que afectan su carrera laboral. La investigación se desarrolla a través del estudio de caso de dos universidades chilenas, mediante el uso de metodología cualitativa se recopila información a través de entrevistas semi-estructuradas con académicas que se desempeñan en cargos directivos universitarios. Los resultados identifican a la conciliación trabajo-familia y la cultura machista como las principales barreras que configuran el techo de cristal dentro de las universidades analizadas.

\section{PALABRAS CLAVE:}

Cargos directivos; Universidades chilenas; Techo de cristal; Responsabilidad social universitaria; Investigación cualitativa.

The phenomenon of the glass roof is analyzed from the perceptions and experiences of the academics regarding the access to the managerial positions in their institutions, materialized in the existence of internal or external barriers that affect the labor career of the women, preventing them from accessing positions of Upper management. The study is developed through a case study of two chilean universities, considering the use of qualitative research methodology gathering information through semi-structured interviews with academics Who hold university positions. The results identify the work-family reconciliation and the macho culture as the main barriers that make up the glass ceiling within the universities analyzed.

\section{KEYWORDS:}

Management positions; Chilean universities; Glass ceiling; University social responsibility; Qualitative research. 
Analisa-se o teto de cristal desde as percepções e experiências das acadêmicas com respeito ao acesso aos cargos diretivos em suas instituições universitárias, materializado em barreiras internas e externas que afetam sua carreira laboral.

A pesquisa se desenvolve através de um estudo de caso de duas universidades chilenas, considerando o uso da metodologia qualitativa reunindo a informação através de entrevistas semiestruturadas com acadêmicas que se desempenham em cargos diretivos universitários. Os resultados identificam a conciliação trabalho-família e a cultura machista como as principais barreiras que configuram o teto de cristal dentro das universidades.

\section{PALAVRAS CHAVES:}

Cargos diretivos; Universidades chilena; Teto de cristal; Responsabilidade social universitária; Pesquisa qualitativa. 


\section{Introducción}

El acceso de las mujeres a los cargos directivos es una problemática estudiada en la literatura especializada desde mediados de la década de los ochenta en los EE.UU. El denominado «Techo de Cristal» (TC) describe las barreras u obstáculos, mayoritariamente invisibles, que las mujeres y afroamericanos deben enfrentar en su carrera laboral, impidiéndoles el acceso a los principales cargos directivos en sus organizaciones laborales.

El TC es una temática interesante de investigar con el propósito de disminuir la discriminación laboral por cuestiones de género en un ámbito menos estudiado, como el del acceso a los cargos directivos, retroalimentando de manera interesante a las políticas públicas que se implementan para garantizar un trato igualitario en el mundo laboral.

Así, en el presente trabajo se exponen resultados del estudio desarrollado con un enfoque cualitativo de investigación, para analizar el acceso a los cargos de dirección superior desde la experiencia, conocimientos y percepciones de diez (10) directivas universitarias de dos universidades de la ciudad de Antofagasta en Chile, se utilizaron entrevistas semi-estructuradas y procedimientos de la Teoría Fundamentada para identificar las categorías, subcategorías y tópicos del TC en las transcripciones de las entrevistas.

En general, entre las entrevistadas no existe un consenso respecto de la configuración del TC en sus universidades, en cuanto a la existencia de barreras que impiden el acceso a los cargos directivos, pero se identifica la crianza y cuidado de los hijos como un impedimento importante.

Así, en la primera parte del artículo se analizan aspectos destacados en la literatura especializada en el TC, fundamentalmente los estudios que analizan dicha temática en el ámbito universitario. En la segunda parte, se presentan los principales resultados y conclusiones obtenidos del análisis de las transcripciones de las entrevistas realizadas.

\section{Revisión de la literatura}

El término TC fue utilizado por primera vez en los EE.UU., en un artículo publicado en The Wall Street Jornal en 1986, para describir un conjunto de barreras invisibles que impiden el acceso de las mujeres a los cargos directivos de las organizaciones laborales (Gaete, 2015; Jackson y O'Callaghan, 2009; Jarmon, 2014). El estudio de las trayectorias profesionales y el acceso de las mujeres a los cargos directivos muestra 
un crecimiento importante en la literatura científica de las últimas décadas, existen diversas hipótesis o líneas de investigación al respecto, entre las que se encuentra el TC (Agut y Martín, 2007; Selva et al, 2011).

Según Matus y Gallego (2015) el TC es una barrera que afecta la carrera laboral de las mujeres, caracterizada por ser:

Invisible por cuanto no es percibida como tal por parte importante de la sociedad y porque no existen impedimentos explícitos discriminatorios contra las mujeres, sino que es mayoritariamente producto de determinados comportamientos sociales. Es acotada puesto que es relativamente fácil determinar en qué punto de la escalera profesional se produce este quiebre. Una especie de umbral sólido que impide romperlo (612).

Asimismo, el TC se transforma en una problemática que en muchas ocasiones no es percibida con facilidad dentro de las instituciones, lo que hace que diferentes tipos de barreras u obstáculos a la carrera laboral de las mujeres se vayan consolidando al interior de las organizaciones laborales, tales como: personales de las propias mujeres, institucionales y sociales (Gaete, 2015; Jarmon, 2014; Martínez y Bivort, 2014). Así, estudios como el desarrollado por Diez (2016) en instituciones escolares españolas concluyen que una de las principales barreras que impide acceder a las mujeres a los puestos de dirección en ese tipo de instituciones, se relacionan con la existencia de una «cosmovisión patriarcal» dominante en la sociedad y cultura española.

De acuerdo con Gaete: el TC «describe cómo la carrera laboral de las mujeres se ve estancada en un nivel jerárquico medio o bajo, sin poder acceder a los principales puestos de trabajo de alta dirección responsables de la toma de decisiones más importantes» (2015: 10), planteando desafíos importantes para las organizaciones laborales al intentar prevenir o eliminar aquellas barreras que puedan afectar las posibilidades de las mujeres de acceder a los puestos directivos en igualdad de condiciones con los hombres.

La consideración del TC como un conjunto de barreras internas o externas a la organización es frecuente en la literatura que analiza dicha temática (Gaete, 2015; Jackson y O'Callaghan, 2009; Jarmon, 2014; Tomás y Guillamón, 2009). En términos generales, las barreras internas describen aspectos que son propios de cada organización y que facilitan el desarrollo del TC, por ejemplo el clima organizacional o las políticas de promoción. En cambio, las barreras externas que generan el TC se relacionan con elementos o condiciones que se generan en cada sociedad o territorio donde está inserta una organización, como por ejemplo la existencia de una cultura patriarcal o el escaso desarrollo del mercado laboral.

A pesar de que el TC es una problemática de investigación analizada desde mediados de la década de los 8o, en el ámbito de la educación superior su estudio 
muestra un desarrollo menor en cuanto al número de investigaciones y publicaciones relacionadas con el análisis de dicha temática en el quehacer universitario (Jarmon, 2014; Matus y Gallego, 2015). Sin embargo, en Gaete (2015) se reseñan algunas investigaciones sobre el TC en universidades de EE.UU. y Europa en la última década, predominando la identificación de barreras internas y externas que facilitan la configuración del TC en ese tipo de instituciones.

En el cuadro 1, se presenta un resumen del estudio de Timmers et al (2010: 720) donde se identifican tres grandes barreras que explican por qué las académicas tienen una baja presencia en cargos directivos.

CUADRO 1 | Barreras para que las académicas accedan a los cargos directivos

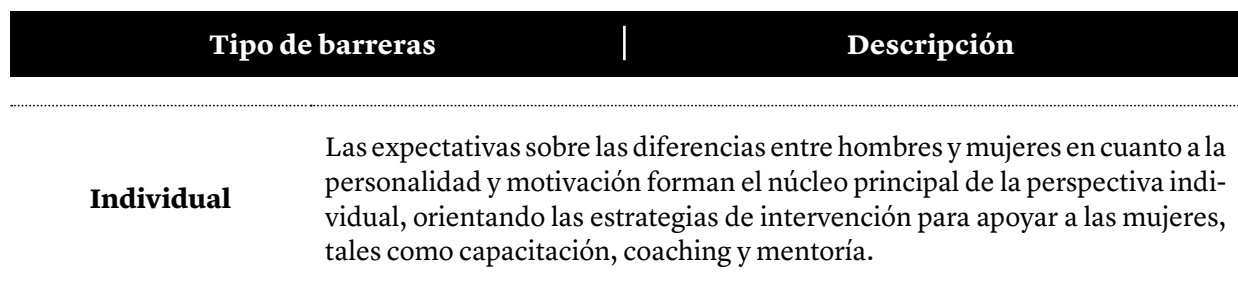

Cultural

Sugiere que la cultura, historia, ideología y políticas organizacionales son elementos relevantes para explicar el limitado éxito de las mujeres en alcanzar posiciones de alto nivel dentro de la organización.

Estructural o

La jerarquía de la organización laboral influye en el ingreso y promoción de Institucional las mujeres, más que los roles individuales o el género.

Fuente: elaboración propia, basado en Timmers et al (2010: 720)

Asimismo, Folch y Ramos (2009) analizan el caso de cuatro universidades de Cataluña, en España, concluyen que entre las barreras y obstáculos que más afectan el acceso de las mujeres a los cargos directivos de las instituciones estudiadas se encuentran las de tipo interna, tales como la baja autoestima, o las barreras externas como la cooptación.

A nivel latinoamericano, Moncayo y Zuluaga (2015) reconocen que cuando se analiza el acceso de las académicas a los cargos directivos universitarios, además del TC como fenómeno explicativo destacan la importancia del denominado «Firewall», es decir, aquellos factores que facilitan o deniegan el acceso. 
\begin{tabular}{l|l} 
CUADRO 2 & Investigaciones sobre acceso de las mujeres a los cargos directivos
\end{tabular} en universidades chilenas.

\section{Autor(es) | Aspectos metodológicos | Principales conclusiones}

Berríos (2007) Estudio cualitativo.

La universidad reproduce el acceso al

Análisis cuantitativo en base a datos poder de manera similar a lo que ocurre en la sociedad, mediante una fuerte estructura patriarcal y androcéntrica tradicional, afectando la carrera laboral de las académicas. sidad estatal.

Baja presencia femenina en los cargos directivos superiores que incluye rectoría, vicerrectoría y, en general, la plana directiva de investigación y posgrados.

La estructuración patriarcal de las uni-

Estudio cualitativo con entrevistas en Martínezy profundidad a 5 académicas de univerBivort, (2014) sidades del sur de Chile pertenecientes al CRUCH.

Análisis cuantitativo en base a datos Gaete (2015) secundarios, de las 16 universidades estatales chilenas. versidades reordena espacio-temporalmente a las mujeres según la misma lógica que históricamente las hacía permanecer en el espacio privado.

Las académicas de dichas universidades tienen escaso acceso a los cargos de elección directa como Rector o Decano, existiendo mayor presencia en cargos de confianza a nivel de Vicerrectorías o Secretarías Docentes.

Fuente: elaboración propia.

En cuanto al caso chileno, el escaso acceso de las mujeres a los cargos directivos en las universidades no es una novedad y está fuertemente presente en el sistema universitario actual, generado a partir de la existencia de un «círculo vicioso» relacionado con el poder dentro de las universidades. Según Montecinos (2001), esto implica que:

La mayoría de las mujeres académicas se concentran en las categorías de profesoras asistentes e instructoras, las categorías más bajas dentro de la jerarquía académica, las con menores ingresos, menor prestigio y también con muy pocas posibilidades de ser elegidas para los cargos directivos que tienen como requisitos ser al menos profesor asociado [...] no poseen ni el poder ni las prerrogativas de estar en una jerarquía académica alta, y cuando lo están, es decir cuando poseen el poder es en carreras eminentemente feminizadas como Humanidades y Salud (235). 
Desde una perspectiva sociológica, el círculo vicioso descrito anteriormente es analizado por Merton (1968), quien plantea la existencia del «Efecto Mateo», en alusión a lo que señala el Evangelio de Mateo 13: 12: «Porque a cualquiera que tiene, se le dará, y tendrá más; pero al que no tiene, aun lo que tiene le será quitado». Con esta analogía bíblica, Merton establece que los investigadores más jóvenes y menos reconocidos dentro de las universidades son despojados de cualquier cuota de poder, en beneficio de aquellos investigadores más consagrados y que mantienen un mayor acceso a los recursos económicos, reconocimiento y posiciones de mayor privilegio dentro de sus universidades y dentro de su respectivo campo científico.

En el Cuadro 2, a pesar del tiempo transcurrido entre las diferentes investigaciones, sigue constante el escaso acceso de las académicas en puestos de alta dirección en las universidades chilenas, predominando una cultura patriarcal y machista bastante marcada como una de las conclusiones transversales de los estudios e investigaciones aludidas.

\section{Metodología}

El objetivo de la investigación es comprender el fenómeno del TC desde las percepciones y experiencias de las académicas de las universidades de la ciudad de Antofagasta respecto del acceso de las mujeres a los cargos directivos de sus instituciones, considerando el paradigma interpretativo-hermenéutico a través de un estudio de

$$
\begin{array}{l|l}
\text { CUADRO }_{3} & \begin{array}{l}
\text { Distribución actual de cargos directivos según género en } \\
\text { universidades estudiadas }
\end{array}
\end{array}
$$

\begin{tabular}{|c|c|c|c|c|}
\hline \multirow[t]{2}{*}{$\begin{array}{l}\text { Cargos directivos/ } \\
\text { distribución por género }\end{array}$} & \multicolumn{2}{|c|}{$\begin{array}{l}\text { Universidad Católica del } \\
\text { Norte (fundada en 1956, } \\
\text { institución privada) }\end{array}$} & \multicolumn{2}{|c|}{$\begin{array}{c}\text { Universidad de Antofagasta } \\
\text { (creada en 1981, institución } \\
\text { estatal) }\end{array}$} \\
\hline & HOMBRE & MUJER & HOMBRE & MUJER \\
\hline Rector & 1 & $\mathrm{O}$ & 1 & $\mathrm{O}$ \\
\hline Vicerrectores & 2 & 2 & 1 & 2 \\
\hline Decanos de Facultades & 7 & 1 & 7 & 1 \\
\hline $\begin{array}{c}\text { Directores de } \\
\text { Departamento }\end{array}$ & 18 & 5 & 18 & 4 \\
\hline
\end{tabular}


caso, aplicando dos criterios de elección de las universidades analizadas: pertenecer al Consejo de Rectores de las Universidades Chilenas (CRUCH) y tener su Casa Central en la ciudad de Antofagasta. El cuadro 3 resume características distintivas de cada universidad estudiada en lo relativo a la distribución de los cargos directivos según género, observándose claramente una mayor presencia de los hombres en ese tipo de puestos.

La técnica de recolección de la información es la entrevista semi-estructurada, para obtener las referencias discursivas acerca del significado que otorgan las académicas de las universidades estudiadas al concepto de TC, no para cuantificar o recoger datos sino para observar y comprender este fenómeno social, a través de la mirada y los sentimientos de las entrevistadas. Las entrevistas se desarrollaron en un formato individual, en un solo encuentro y enfocadas en una sola temática. El entrevistador proporcionó el mismo estímulo o planteamiento inicial a cada entrevistado sobre la problemática estudiada, utilizándose una pauta de cotejo con los tópicos relevantes para la investigación, presentados en el Cuadro 4.

CUADRO 4 Pauta de cotejo de las entrevistas realizadas durante la investigación

$\begin{array}{cl}\text { Encabezado de la } & \text { Junto con agradecer su buena disposición al conceder esta entrevista, quisiera } \\ \text { señalar que el objetivo de la investigación es «comprender el fenómeno del } \\ \text { entrevista }\end{array} \quad \begin{aligned} & \text { techo de cristal desde las percepciones y experiencias de las académicas de } \\ & \text { las universidades de la ciudad de Antofagasta, respecto del acceso de las } \\ & \text { mujeres a los cargos directivos de sus instituciones», para lo cual le propo- } \\ & \text { nemos las siguientes preguntas. }\end{aligned}$

Tópico

Preguntas

¿Cómo ha sido su experiencia en el ejercicio de cargos directivos en los que se ha desempeñado en la universidad?

Experiencia en ¿Cuáles son los aspectos positivos y negativos que se generan para una mujer cargos directivos en el ejercicio de un cargo directivo dentro de la universidad?

¿Cuáles son las principales dificultades u obstáculos que ha encontrado al desempeñar un cargo directivo en la universidad?

¿Cuáles serían las formas de expresión que permiten observar la existencia del TC en la universidad?

Percepción sobre el ¿Cuáles serían las principales causas que favorecen la configuración del TC techo de cristal en la universidad?

¿Qué medidas, estrategias o acciones propondría para eliminar el TC dentro de la universidad? 
En cuanto a los informantes clave, se considera el concepto de muestra estructural o teórica, es decir, aquella que prioriza la representatividad sustantiva y no estadística, porque se busca incluir a los actores sociales de interés en cuanto al discurso que reproducen, buscando saturar los lugares de enunciación de su relato. Por ello, la muestra del estudio busca favorecer la calidad del discurso en base a la experiencia de los informantes clave y no la representación estadística de sujetos a incluir en el estudio, considerando a las académicas de ambas universidades que tengan experiencia en cargos directivos.

Para el análisis de las transcripciones se consideran las bases y procedimientos propuestos por la Teoría Fundamentada, mediante una perspectiva narrativa, elaborando un razonamiento por parte del investigador, apoyado y explicado seguidamente mediante diferentes fragmentos extraídos de las entrevistas realizadas durante la investigación para identificar los significados que los actores sociales asignan a la problemática estudiada. Los incidentes recogidos en cada entrevista fueron anonimizados y serán identificados con la letra $\mathrm{E}$ y el número correspondiente a la entrevista realizada, para garantizar la confidencialidad de la identidad de las entrevistadas, comprometida mediante la firma de un consentimiento informado con cada una de ellas.

Asimismo, se siguieron los procedimientos establecidos por la teoría fundamentada de los datos (Grounded Theory) que se caracteriza por la generación o surgimiento de teoría a partir de los datos proporcionados por los propios sujetos sociales, con lo que se intenta explicar los fenómenos o procesos sociales estudiados en una investigación, mediante el siguiente procedimiento: 1) identificación de incidentes para asignarle códigos (microanálisis); 2) comparación de los códigos sustantivos, conceptos y categorías (Codificación); y 3) la integración de estos elementos en una teoría que se elabora a partir de los datos que permitan construir hipótesis teóricas.

\section{Resultados}

A partir de las entrevistas realizadas con diferentes académicas que ocupan cargos directivos en universidades de la ciudad de Antofagasta, es posible observar en el Cuadro 5 la teoría sustantiva generada a partir de la aplicación de los procedimientos de la Teoría Fundamentada, expresado en diferentes categorías, subcategorías y códigos.

El Cuadro 5, resume los principales hallazgos obtenidos en las entrevistadas realizadas identificando tres grandes barreras para el caso estudiado que las académicas deben enfrentar para acceder a los cargos directivos en sus universidades. 
CUADRO 5 Categorías y subcategorías del concepto de TC

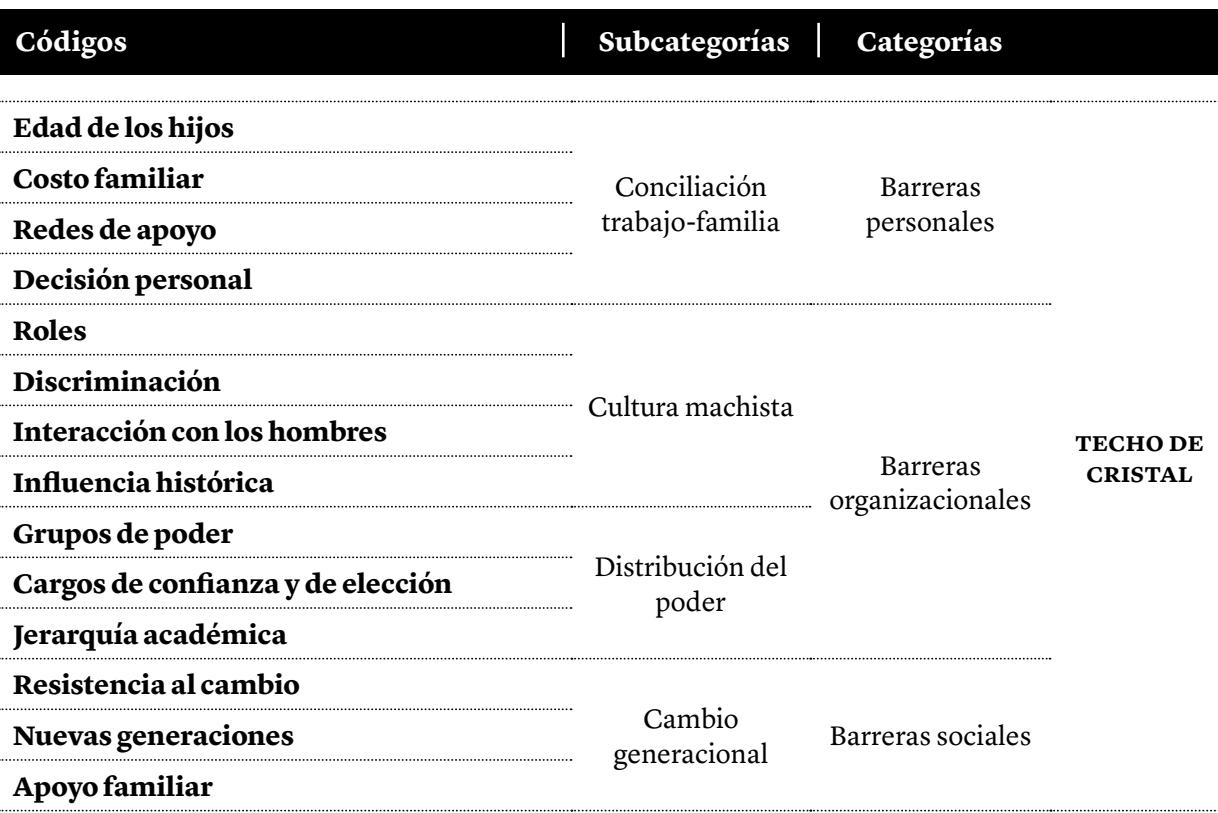

\section{Manifestaciones del Techo de Cristal}

En primer lugar, el TC se manifiesta con mayor claridad en dos de los cargos directivos más importantes dentro de las universidades: rectores y decanos. En el caso de los rectores, algunas entrevistadas dan cuenta del hecho de que nunca una mujer ha sido rectora en las universidades del CRUCH:

Si tú miras la [Universidad] Diego Portales, te guste o no te guste, hay una líder potente femenina, entonces ha habido Rectoras y en el CRUCH [...] no ha habido ninguna Rectora, entonces también ahí marca en el ámbito privado (E5).

La mejor prueba es que no tienes ni Rectores mujeres, entiendes y yo pienso que si tú haces un cedazo [criba] y miras cuantas mujeres trabajan en la universidad y cuantos hombres trabajan debe estar equiparado y tú no te vas a encontrar con una mujer a cargo de una universidad (E8).

Otro espacio donde las entrevistadas observan la configuración del TC en las universidades es en el cargo de Decano: 
Sitú miras las decanaturas, salvo decanatura en Educación donde mayoritariamente hay más mujeres, si lo sacamos y miramos la Ingeniería todas están en manos de hombres, o sea en el área de salud que yo creo que tiene el 70\% mujeres, ¿cuantas decanas ha habido? que yo sepa nunca una decana, siempre han sido hombres (E8).

Tuve la oportunidad la semana pasada de asistir a la primera reunión de los decanos [...] ese consejo reúne veintiún decanos, de los cuales cinco son mujeres y ahora ultimo porque yo tengo entendido que hasta hace dos años atrás solamente habían dos mujeres, entonces es cierto que en los cargos directivos hay una presencia menor de mujeres (E9).

Sin embargo, el TC también afecta a otros cargos directivos de exclusiva confianza o de carácter colegiado, tales como vicerrectorías o junta directiva respectivamente:

Cuando escuchaba en otra reunión a la Vicerrectora Académica de la [Universidad de] Chile: ¡hasta Vicerrectora no más, inteligentísima!, colegas aquí que yo tengo inteligentísimas pero tenemos un techo [...] y no sé si el techo es autoimpuesto, porque no nos hemos atrevido, no sé, ¿o es tan fuerte la cultura? (E5).

Que te elijan para llegar a la Junta como mujer también es bien difícil, piensa tú que somos del año 81, que tenemos Junta Directiva a la fecha ¿ha habido cuántas mujeres? han sido 3 [...] no ha habido más mujeres en la Junta Directiva como representante de los académicos (E8).

Así, para nuestras entrevistadas el TC en las universidades estudiadas obedece a la existencia de tres grandes tipos de barreras: personales, organizacionales y sociales. Estos obstáculos se transforman en razones importantes para desistir de ocupar cargos directivos o les dificultan su permanencia en los mismos.

En la Figura 1, se describen los tipos de barreras identificadas en el estudio, coincidiendo con Timmers et al (2010: 720) en cuanto a la existencia de barreras individuales, culturales e institucionales. Otras investigaciones similares evidencian mayor preponderancia al análisis de las barreras de tipo organizacional o social (Berríos, 2007; Kiss et al, 2007; Martínez y Bivort, 2014).

\section{Barreras personales}

Según nuestras entrevistadas, el eje principal de las barreras personales, descrito en la Figura 2, está ubicado en la maternidad y las posibilidades que las académicas encuentran dentro de sus universidades para poder conciliar el trabajo con la familia, especialmente en cuanto a la mayor dedicación de tiempo que implican las responsabilidades asociadas a los cargos directivos. 
FIGURA 1 | Barreras que constituyen el TC en las organizaciones

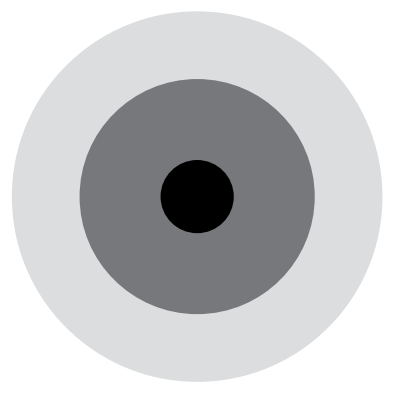

- Barreras personales:

Aspectos vinculados con la vida personal o familiar de las mujeres, que afectan o condicionan sus propias opciones de acceder a los cargos directivos.

Barreras organizacionales:

Elementos o situaciones que están presentes en la organización en la que las mujeres desarrollan su carrera laboral, que obstaculizan su acceso a los cargos directivos.

\section{Barreras sociales:}

aspectos distintivos de la cultura propia de la sociedad o el territorio donde funciona la organización laboral.

FIGURA 2 Aspectos relevantes para conciliar trabajo y familia

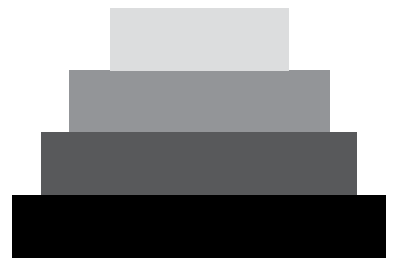

Voluntad propia para conciliar

Redes de apoyo

Costo familiar

Edad de los hijos

Fuente: elaboración propia .

Un primer aspecto que destacan las entrevistadas sobre la conciliación trabajo-familia tiene relación con la edad de los hijos, porque cuando estos se encuentran más pequeños la dificultad para desempeñarse en cargos directivos aumenta:

Influye bastante la etapa de la vida que uno se encuentre, yo siento que efectivamente asumir un cargo a nivel de Vicerrector o Rector con una familia donde los hijos son muy pequeños, yo creo que las mujeres optamos por dar un paso al costado, para nosotras yo pienso que la demanda de nuestros hijos cuando son pequeños es alta, hay que ayudarlos con las tareas, están las reuniones, etc (E1). 
Mucho tiempo yo no me involucré más porque tenía a los niños más chicos [pequeños], entonces uno sabe que no puede rendir y no te puedes comprometer con niños chicos, en cambio con niños más grandes uno sabe que se desliga un poco más y puede dedicarse más a su trabajo (E2).

A partir de las responsabilidades familiares propias de la maternidad, algunas académicas entrevistadas identifican la existencia de un «costo familiar» importante asociado a la asunción de cargos directivos:

Como mujer siento que son cargos muy demandantes y por lo tanto el costo familiar no es menor y quizás eso mismo lleva a que las mujeres tengamos, esto es una opinión, no andamos buscando acceder a estos cargos (E1).

Optaste por aceptar esta invitación de ser Vicerrector, claro esto podría ser una carrera, sabes que a mí me interesa mantenerme como Vicerrector y de ahí a la Rectoría, el universo juna cosa así!, la verdad es que el costo familiar todo el tiempo me hace cuestionarme esta decisión (E4).

Para enfrentar de mejor manera los requerimientos de conciliación trabajo-familia, las entrevistadas reconocen la importancia de contar con «redes de apoyo»:

Siento que ahora además que los hombres también tienen que asumir los roles, entonces con mayor razón si uno tiene un cargo tiene que tener el apoyo de la familia en ese sentido (E6).

La gente joven por ejemplo, para ellas sí que creo que el hecho de tener su casa y sus hijos es complejo, porque no vivieron en la misma época que lamentablemente me tocó vivir a mí, en mí época nosotros teníamos a nuestras mamás, nuestras familias que era un gran apoyo, yo a mi hija nunca la tuve en manos de empleada [doméstica] porque yo tenía mi suegra y mi suegra cumplía el rol de cuidarme a mi hija mientras yo no estaba y en los otros períodos venían mis papás (E8).

Finalmente, un aspecto importante para conciliar trabajo y familia pasa por la propia voluntad o decisión personal de las mujeres por intentar alcanzar dicho equilibrio:

Hasta hace muy poco tiempo trabajaba hasta las tantas aquí y me llevaba pega [trabajo] $\mathrm{pa}[\mathrm{ra}]$ la casa, entonces ahora he tomado decisiones, igual me quedo hasta tarde pero no me llevo nada para la casa el fin de semana [...] pero eso ya es una decisión propia y privilegio la familia, mis sobrinos, mis hermanos o sea sábado y domingo yo trato de desconectarme (E9).

Estos últimos años yo no me llevo nada para la casa para hacer, porque mi tiempo personal es mío y lo que hay que hacer aquí y lo hago en mi tiempo, el problema que 
acá si tú quieres avanzar en el informe poco menos te tienes que esconder, ir a un lugar que te dejen tranquilo (E10).

La barrera personal generada a partir de las dificultades para conciliar trabajo-familia, debiera ser un aspecto tremendamente importante en las políticas de recursos humanos de las universidades, para eliminar efectivamente el TC.

\section{Barreras organizacionales}

En este segundo tipo de barreras, existen dos grandes ámbitos en los cuales se concentran los discursos de las entrevistadas: cultura machista y distribución del poder. En la Figura 3, se observan los elementos incluidos en cada una de las subcategorías.

En ambas subcategorías, el poder dentro de las universidades se transforma en un elemento clave para comprender mejor la configuración del TC en este tipo de instituciones, donde las relaciones de poder en las universidades describe una temática estudiada por la sociología desde hace algunas décadas (Becher, 2001; Bok, 2008; Bourdieu, 2008; Merton, 1968; Ortega y Gasset, 2007; Weber, 2009).

FIGURA 3 Ámbitos de las barreras organizacionales en las universidades
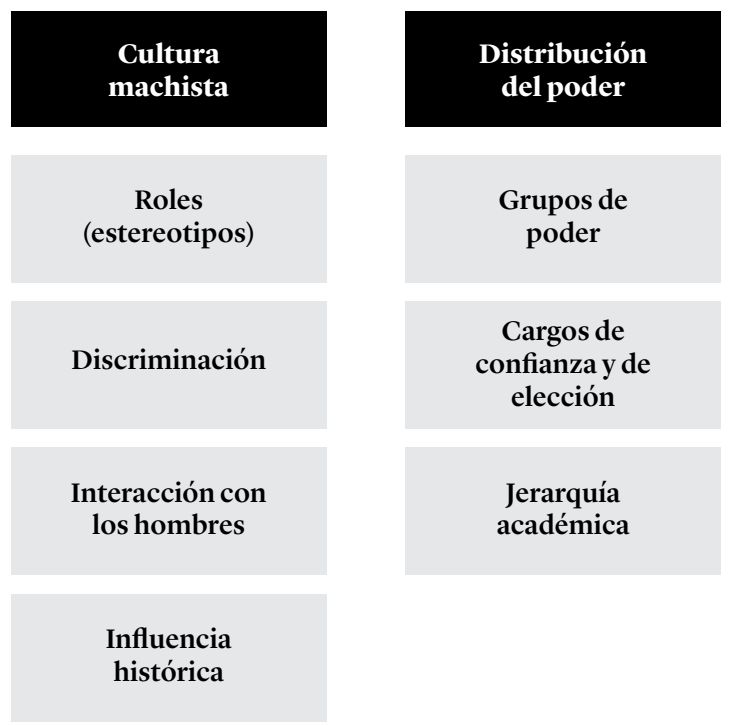


\section{Cultura machista}

Un primer elemento que se observa en la revisión de las entrevistas se relaciona con la existencia de ciertos «estereotipos» que asignan roles específicos a las mujeres:

Aunque tengamos un tremendo trabajo llegamos a la casa y tenemos que cocinar, hacer cosas y ver los niños, bañar las guaguas [bebé] y todas esas cosas que son propias de la maternidad y que uno tampoco las deja de lado, pero yo creo que es en la cultura en nosotros en nuestro inconsciente o subconsciente vendrá eso de que el hombre se tiene que preparar mejor entonces para llegar a estos cargos (E2).

Pero el varón que tiene una mujer que no es profesional, él se lleva el peso en la familia y yo también entiendo que el varón tiene que jugárselas para decir: iyo tengo que llegar al cargo! [...] cuando miro y digo: ¡sí, pero esta persona es el proveedor de su familia, se queda sin pega [Trabajo] es complicado! (E3).

Otro aspecto destacado por las académicas entrevistadas se relaciona con la existencia de un proceso de «discriminación» como consecuencia del TC:

Hay cosas que se me han hecho más difíciles, no por ser mujer, sino porque en cada cargo que tú vas a gestionar algo hay un hombre [...] si tú no entras en ese lenguaje de las representaciones del hombre que está en ese cargo, no tienes las mismas posibilidades $(\mathrm{E} 7)$.

Si una mujer lo hace y se equivoca la crucifica, si un hombre hace lo mismo y se equivoca no es crucificado, para mí es la mejor demostración del machismo puro (E8).

Un tercer aspecto que describe la cultura machista asociada con la existencia del TC, se relaciona con la manera en la cual las mujeres interactúan con los hombres en su trabajo:

Cuando me hablaban a mí de discriminación de género yo no lo alcanzaba a entender, porque yo nunca lo había vivido ¿te das cuenta? Ni siendo estudiante, ni en mis 27 años que estuve en el Departamento, Facultad con los colegas nunca, [...] aquí arriba lo sentí, lo sentí pero no violentamente (E)).

Estudié en una Universidad donde todos eran hombres y después trabajé en una universidad que cuando yo entré a trabajar casi todos eran hombres, éramos pocas las mujeres, habían unas cuatro o cinco en total, éramos poquitas entonces la formación de uno ya es [...] un poco más distinta o diferente. Por eso, a veces los colegas conversan y todo y dicen: ino contigo es diferente conversar, contigo que con el resto de las mujeres!, porque uno tiene la visión un poco más parecida a la del hombre ahora (E8). 
Finalmente, en las transcripciones de las entrevistas existen algunas referencias a lo que se podría identificar como una influencia histórica, la cual favorece la configuración del TC dentro de las universidades estudiadas:

Que en la mayoría de los cargos haya hombres es por nuestra tradición occidental, todo lo que viene del pensamiento griego, o sea en el pensamiento griego no hay mujeres, uno nombra a Pitágoras, Heráclito, los mismos dramaturgos (E7).

La sociedad chilena ha cambiado radicalmente en estos últimos años, pero sigue habiendo ese machismo, que el hombre es el que está en posiciones de toma de poder o decisiones (E9).

Así, las barreras organizacionales que facilitan el TC se configuran a partir de la existencia de diferentes elementos culturales dentro de las universidades, relacionando este fenómeno con la existencia de un patriarcado, similar a lo que establece Diez (2016) en su estudio sobre esta temática.

\section{Distribución del poder}

Según las académicas entrevistadas, entre las barreras organizacionales que pueden configurar el TC dentro de las universidades, un factor muy importante es la manera en la cual se desarrolla la dinámica del poder dentro de las mismas:

Al final hay acuerdos políticos y se forman los grupos que yo creo que no es malo, pero habían mujeres que perfectamente podrían haber competido en igualdad y con mérito, con capacidades ellas dos y no tenían más mal curriculum que el Decano que salió (E6). Me presenté como candidata y la elección la ganó otra persona, pero ahí hubo un problema [...] porque después la persona que salió no pudo ejercer porque tenía una incompatibilidad de orden administrativo, había como dos fuerzas, había pugnas que se disputaban el poder (E9).

Otro aspecto relacionado con la distribución del poder, radica en las razones que influyen en la elección democrática de algunos cargos directivos:

Los cargos al ser votados la gente se lleva sus votantes como los políticos y claro [...] las decisiones no siempre son en bien de la universidad, sino que eso reconozco que es una realidad que existe, entonces ahí se producen vicios (E6).

La mayoría de los colegas pensaban que yo podía ocupar ese cargo, anteriormente había habido varones, y en ese momento yo creo que fue un poco no tanto por el género sino más bien por una cuestión de edad, necesitaban, porque se venían muchas tareas, necesitaban una persona -en ese tiempo- que fuera joven y que pudiera ocupar ese cargo, y yo había trabajado antes con el anterior Director (E9). 
Un tercer elemento que surge de las entrevistas para describir la manera en la cual está distribuido el poder como facilitador del TC se relaciona con la «Jerarquía Académica», como un aspecto distintivo del funcionamiento de los sistemas universitarios:

Creo que en el mundo académico sí tú no les presentas a tus colegas un doctorado, un proyecto con financiamiento externo, la verdad que este puesto como Vicerrector [...] entonces hubiera sido muy difícil (E4).

Por el asunto de la Comisión de Evaluación Superior Académica uno ve que algunas Facultades la posibilidad de ascender es menos compleja que en otras, incluso habría que ver la distribución porcentual entre mujeres versus hombres y en la Facultad que más profesores asistentes hay es aquí y cuesta mucho poder ascender y así se maneja el poder (E1O).

Así, la existencia de grupos de poder y la jerarquía académica influyen en las posibilidades que tienen las académicas de acceder a los cargos directivos de confianza o de elección democrática, transformándose en aspectos importantes a considerar a la hora de implementar estrategias que rompan con la hegemonía masculina dentro de las universidades.

\section{Barreras sociales}

Finalmente, es posible identificar discursos de las académicas relacionados con el cambio generacional que es necesario observar dentro de las universidades, como expresión de una barrera social que favorece la existencia del TC, a partir de la presencia de algunos aspectos identificados en la Figura 4.

Así, un elemento del cambio generacional como barrera social es la resistencia al cambio de los hombres, como un obstáculo para el normal acceso de las académicas a los cargos directivos:

Estamos hablando además de un cambio generacional de estas personas con más de 60 años, no es fácil que una mujer joven te diga: jesto tiene que hacerlo así! (E3). Hay algunos los menos, los menos que les cuesta el tema todavía y es gente más bien mayor y pocos, no es mayoritario, mayoritariamente siento que tenemos un buen vínculo (E4).

Otro aspecto destacado por las académicas entrevistadas respecto del cambio generacional, se relaciona con la forma como las nuevas generaciones comprenden sus roles familiares y laborales: 
FIGURA 4 $\quad$ El cambio generacional como barrera social en las universidades

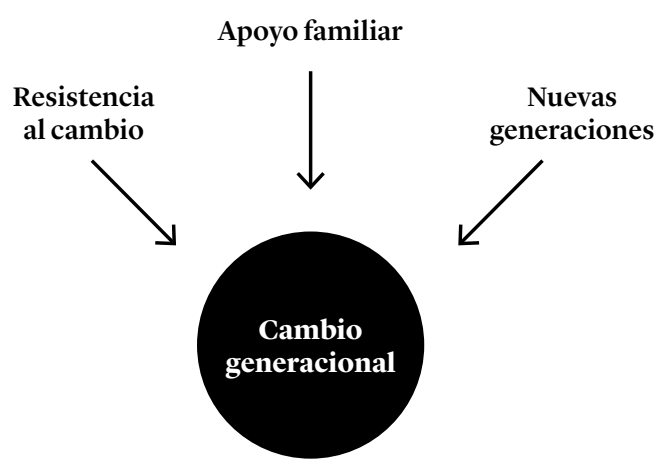

Fuente: elaboración propia .

Yo tengo mujeres, ellas ya vienen con un concepto de pelear de igual a igual, con un cambio generacional porque las mamás de uno eran más de casa yo creo que eso ya cambió las nuevas generaciones ya vienen con el que los dos son papás que tú te quedarás un día, yo el otro, entonces yo me imagino que unos años más las mujeres pelearán más por acceder a cargos (E1).

También las nuevas generaciones vienen de nuestra formación, por ejemplo ya en mi hija yo veo distinto eso, pero otro factor común que yo creo que influye es el interés de las mujeres en interactuar en estos mundos (E7).

Finalmente, el cambio generacional también es relacionado con la importancia del apoyo familiar en diferentes etapas de sus vidas, lo que les ha permitido de una u otra manera desarrollar sus carreras académicas en cargos directivos:

Las generaciones que vienen más atrás están haciendo el cambio en el juego, entonces me desarrollo profesionalmente, tengo mi guagua [bebé], pero al final del día si mi profesión tiene mayor desempeño que la tuya, es el marido el que se está quedando en la casa (E3).

La generación de mi mamá, que es la generación del 45, se esforzó para que sus hijas fueran a la universidad, de hecho yo recuerdo el momento en que mi mamá le decía a mi papá: ¡deja que vaya a la universidad, porque se va a desarrollar!, a pesar de que era un mundo que ella no conocía, ella lo quería para mí y mi papá no quería porque estábamos justo en mucha convulsión, te estoy hablando del $83(\mathrm{E} 7)$. 
Por ello, el cambio generacional está condicionado por los roles que cumplen diferentes actores del grupo familiar de las académicas que ocupan cargos directivos, tanto en el rol que jugaron sus madres en el pasado, como respecto del papel que actualmente deben cumplir sus cónyuges.

\section{Conclusiones y recomendaciones}

La comprensión del TC desde las percepciones y experiencias de las académicas de las universidades estudiadas, permitieron identificar un conjunto de barreras personales, organizacionales y sociales que facilitan que dicha problemática se desarrolle al interior de sus instituciones. Dicho fenómeno es especialmente perceptible para las académicas que desempeñan un cargo de elección democrática, tales como decano o directora de departamento, identificando el TC desde la perspectiva y la experiencia que provee la dinámica del poder al interior de las universidades.

Las barreras personales identifican el dilema que las mujeres deben enfrentar para compatibilizar sus responsabilidades maternales y laborales al asumir un cargo directivo, especialmente cuando sus hijos están pequeños o en edad escolar, debiendo en muchas ocasiones recurrir a las redes familiares o incluso evaluando la posibilidad de postergar el desarrollo de su carrera laboral en cargos directivos. En este ámbito, la conciliación trabajo-familia se transforma en un desafío especialmente relevante para que las universidades estudiadas puedan implementar estrategias y políticas institucionales que respondan a las dificultades que las mujeres enfrentan cuando tienen hijos pequeños, traduciéndose en apoyos más concretos y específicos que disminuyan los costos familiares que pueda implicar la asunción de un cargo directivo de cualquier nivel o naturaleza, evitando que dicha decisión quede condicionada a los apoyos familiares con los que pueda contar cada académica.

Así, pueden considerarse como posibles acciones a implementar: la disponibilidad de permisos adicionales a los estipulados legalmente, horarios de trabajo flexibles o modalidades de teletrabajo, las que pueden transformarse en medidas de conciliación que permitan a las mujeres enfrentar de mejor manera los requerimientos de la maternidad relacionados con aspectos tales como la lactancia, enfermedades de los hijos o asuntos escolares.

En lo relativo a las barreras organizacionales, estas se relacionan fundamentalmente con aspectos culturales y de relaciones de poder al interior de las universidades analizadas. Así, adquiere especial relevancia la fuerte tradición histórica que tienen los estereotipos machistas dentro de las universidades, expresados en una bajísima participación de las mujeres en los cargos directivos de ambas universidades, espe- 
cialmente a nivel de decanos y directores de departamentos, como se describe en el cuadro 3 de este trabajo, donde ambos tipos de puestos de trabajo son de elección democrática directa configurándose la segunda subcategoría de este tipo de barrera: la distribución del poder.

Dentro de las acciones recomendadas para superar este tipo de barreras en las universidades, debieran implementarse procesos de gestión del cambio que permitan modificar la cultura organizacional en cuanto a la existencia de ámbitos afectados por alguna diferenciación o discriminación de roles que generen estereotipos que afecten a las mujeres. También, las modificaciones a la estructura organizacional que establece la jerarquía y el poder al interior de las instituciones universitarias, que eviten la consolidación de grupos de poder para el acceso a los cargos de confianza o de elección democrática, o su influencia en la determinación de la jerarquía académica de las mujeres.

Finalmente, en lo relativo a las barreras sociales para que las mujeres accedan a los cargos directivos al interior de las instituciones estudiadas, predomina en los discursos de las entrevistadas la importancia del cambio generacional dentro de las universidades, para eliminar importantes enclaves de resistencia al cambio avanzando más rápidamente en la incorporación efectiva de valores sociales de importancia, tales como la inclusión social o la no discriminación, que en el caso de las nuevas generaciones ya comienzan a evidenciar su incorporación al momento de desarrollar su carrera laboral, especialmente en lo relativo al apoyo familiar que es necesario entre ambos cónyuges al momento de compartir las responsabilidades laborales y familiares, que permitan a las mujeres extender su desarrollo profesional hacia cargos directivos.

Así, debido a que la sociedad chilena se encuentra inmersa en un proceso de cambio social y generacional de importancia, dentro de las acciones que las instituciones estudiadas pueden implementar para adecuarse a dicho contexto social, la implementación de una gestión de recursos humanos que incorpore criterios de diversidad demográfica y cognitiva a la hora de acceder a los cargos directivos y en general en el desarrollo de la carrera laboral de los miembros de la organización. Lo anterior, puede permitir la implementación de políticas de renovación de plantas académicas, fortalecimiento de los procesos de concursabilidad por mérito para los cargos directivos o el diseño de puestos de trabajo de nivel directivo más acorde con los requerimientos de una sociedad en pleno proceso de cambio social, adaptando a las universidades estudiadas a un nuevo contexto social y organizacional. 


\section{Referencias}

AGUT, S. y MARTÍN, P. (2007). Factores que dificultan el acceso de las mujeres a puestos de responsabilidad: una revisión teórica. Apuntes de Psicología, 25 (2), 201-214.

BECHER, T. (2001). Tribus y territorios académicos. La indagación intelectual y las culturas de las disciplinas. Barcelona: Gedisa.

BERRÍOS, P. (2007). Análisis sobre las profesoras universitarias y desafíos para la profesión académica en Chile. Revista Calidad en la Educación, (26), 39-53.

BOK, D. (2008). Más allá de la torre de marfil. La responsabilidad social de la universidad moderna. Buenos Aires: Universidad de Palermo.

BOURDIEU, P. (2008). Homo Academicus. Madrid: Siglo XXI.

DIEZ, E. (2016). Female principals in Education: Breaking the Glass Ceiling in Spain. Paidéia, 26 (65), 343-350.

GAETE, R. (2015). El techo de cristal en las universidades estatales chilenas. Un análisis exploratorio. Revista Iberoamericana de Educación Superior, 6 (17), 3-20.

FOLCH, M. y RAMOS, C. (2009). Las barreras y los obstáculos en el acceso de las profesoras universitarias a los cargos de gestión académica. Revista de Educación, (350), 253-275.

JACKSON, J. y O'CALLAGHAN, E. (2009). What do we know about glass ceiling effects? A taxonomy and critical review to inform higher education research. Research in Higher Education, 50, $460-482$.

JARMON, L. (2014). «Cracking the glass ceiling: A phenomenological study of women administrators in higher education». Graduate Theses and Dissertations, Iowa State University, digital repository paper 13789. Consultado [o4 de noviembre de 2016] Disponible en: http:// lib.dr.iastate.edu/cgi/viewcontent.cgi article $=4796 \&$ context $=$ etd

KISS, D., BARRIOS, O. y ÁLVAREZ, J. (2007). Inequidad y diferencia. Mujeres y desarrollo académico. Estudos Feministas Florianópolis, 15 (1), 85-105.

MARTÍNEZ, S. y BIVORT, B. (2014). Procesos de producción de subjetividad de género en el trabajo académico: Tiempos y espacios desde cuerpos femeninos. Psicoperspectivas: Individuo y sociedad, 13 (1), 15-22.

MATUS, M. y GALLEGO, N. (2015). Techo de cristal en la Universidad. Si no lo veo no lo creo. Revista Complutense de Educación, 26 (3), 611-626.

MERTON, R. (1968). The Matthew Effect in Science. The reward and communication systems of science are considered. Science, 159 (3810), 56-63. 
MONCAYO, B. y ZULUAGA, D. (2015). Liderazgo y género: barreras de mujeres directivas en la academia. Pensamiento y Gestión, (39), 142-177.

MONTECINOS, S. (2001). Diversidad y desigualdad en el debate público chileno. En Riveros, Luis (Ed.) La Universidad piensa a Chile. Anales de un Foro académico (pp.227-240). Santiago de Chile: LOM Ediciones.

ORTEGA y GASSET, J. (2007). Misión de la Universidad. Madrid: Biblioteca Nueva.

SELVA, C., SAHAGÚN, M. y PALLARÉS, S. (2011). Estudios sobre trayectoria profesional y acceso de la mujer a cargos directivos: un análisis bibliométrico. Revista de Psicología del Trabajoy de las Organizaciones, 27(3), 227-242.

TIMMERS, T., WILLEMSEN, T. y TIJDENS, K. (2010). Gender diversity policies in universities: a multi-perspective framework of policy measures. Higher Education, (59), 719-735.

TOMÁS, M. y GUILLAMÓN, C. (2009). Las barreras y los obstáculos en el acceso de las profesoras universitarias a los cargos de gestión académica. Revista de Educación, (350), 253-275.

WEBER, MAX (2009). La ciencia como profesión. Madrid: Biblioteca Nueva. 